\title{
Rechtsgeschichte
}

www.rg.mpg.de

http://www.rg-rechtsgeschichte.de/rg14

Zitiervorschlag: Rechtsgeschichte Rg 14 (2009)

Rg 142009

$217-219$

http://dx.doi.org/10.12946/rg14/217-219

\section{Stefan Kroll}

Interkultureller Transfer: Zur Übersetzung des Völkerrechts 
und interessante Modelle entwickelt. Aus der Sicht des geltenden Rechts ist schließlich zu bedauern, dass die Darstellung - nicht durchgängig, aber weithin - mit dem Jahr I970 abschließt; damit bleibt der Übergang zu neueren und aktuellen Erscheinungen - dem Streit um eine "Wirtschaftsverfassung ", der europäischen Integration, dem wachsenden Einfluss des amerikanischen Rechts - unberücksichtigt.

All das kann den Gesamteindruck nur marginal trüben. Mathias Schmoeckel ist ein vorzüglich konzipiertes und dokumentiertes Werk zu verdanken, das nicht nur den Studierenden, sondern auch der wissenschaftlichen Arbeit den Zugang zur Entwicklung der Regelung der wirtschaftlichen Abläufe und das Verständnis ihrer komplexen Zusammenhänge wesentlich zu erleichtern vermag. Die verbleibenden Eierschalen seiner Entstehungsgeschichte können unschwer abgestreift werden, wenn sich Autor und Verlag - hoffentlich bald - zu einer zweiten Auflage entschließen.

Friedrich Kübler

\section{Interkultureller Transfer: Zur Übersetzung des Völkerrechts*}

In China war das europäische Völkerrecht bis zur Mitte des I9. Jahrhunderts weitgehend unbekannt. Abgesehen von einzelnen Verträgen aus dem I7. und I 8. Jahrhundert gab es weder Vertragspraxis noch Völkerrechtswissenschaft. Dies änderte sich erst durch Chinas Kriege mit westlichen Mächten. Die »Barbaren « nutzten ihre militärische Überlegenheit gegenüber China, um durch erzwungene völkerrechtliche Friedensverträge Handelsinteressen zu verwirklichen und halbkoloniale Strukturen zu etablieren. Diese Friedensübereinkommen wurden und werden, als »ungleiche Verträge « überschrieben, vielfach in der wissenschaftlichen Literatur besprochen.

Weniger Interesse galt bislang dem eigentlichen Aneignungsprozess des Völkerrechts in China - »Translation, Reception and Discourse«-, der durch die ungleichen Verträge ausgelöst wurde. Dieser Frage widmet sich der norwegische Sinologe Rune Svarverud. Sein Ziel ist es, die Aneignung des Völkerrechts in China als indigene Leistung verständlich $\mathrm{zu}$ machen und sie nicht lediglich als Reaktion auf westliche Einflüsse darzustellen. Dies ist nicht zuletzt als Kritik an der Tradition des Sinologen John K. Fairbank (I907-I99I) zu verstehen, der die chinesische Modernisierung in seinen frühen Werken als Antwort auf die Herausforderung des Westens interpretierte (Impact-Response-Model) und hierdurch die historiographische Sinologie des 20. Jahrhunderts maßgeblich prägte.

Hieran anknüpfend und darüber hinaus hinterfragt Svarverud das Verständnis des Tributsystems in der allgemeinen Geschichtswissenschaft. Das Tributsystem ging dem Völkerrecht

\footnotetext{
* Rune Svarverud, International Law as World Order in Late Imperial China. Translation, Reception and Discourse, I847-I9II, Leiden: Brill 2007, 322 S., ISBN 978-90-04-I60I9-4
} 
als Ordnung der internationalen Beziehungen in Ostasien voraus. Es dient in der Erforschung der Spätphase der Qing Dynastie meist als Referenz, an der sich die umfassende Dimension des Transformationsprozesses offenbart. Kontrastiert mit dem Völkerrecht, wird das Tributsystem als rückständig interpretiert - auch im zeitgenössischen Kontext. Anhand des Vergleichs der beiden Ordnungen wird die Modernisierungsleistung, von wem auch immer sie ausging, besonders transparent. Svarverud sieht in der Gegenüberstellung der sich gegenseitig ausschließenden Ordnungen eine vereinfachte Dichotomie (»simplistic idea of a dichotomy«), welche die dahinter liegenden komplexeren $\mathrm{Zu}$ sammenhänge der Transformation verschleiere.

So anregend diese Abgrenzungen sind, sie überzeugen letztlich beide nicht. Dies vor allem deshalb, weil auch der Autor sie nicht aufrecht zu erhalten vermag. Die allgemeine Kritik an der Verwendung des Tributsystems als kontrastierenden Elements wird von Svarverud selbst relativiert. "For our case it may serve as a system of reference and as a framework for understanding the context of the introduction of bilateral treaties [...] and as an intellectual framework forming the background for the introduction of international law [...]." Ausgerechnet für die Heuristik der eigenen Untersuchung erkennt Svarverud also einen Gebrauchswert in der Gegenüberstellung.

Die Ablehnung der Impact-Response-Hypothese wird durch das Buch in seiner Gesamtaussage in Frage gestellt. Svarveruds Analysen lesen sich als eine Argumentation für diese These, und nicht als deren Widerlegung. Gerade in den Passagen, in denen der Autor Ansätze einer aufkeimenden chinesischen Völkerrechtswissenschaft beschreibt, wird der Einfluss westlicher Vorlagen besonders deutlich (I33-I5O). Auch die Rekonstruktion der Übersetzungen völkerrechtswissenschaftlicher Texte verstärkt diesen Eindruck (69-150). So wären diese, wie Svarverud folgert, ohne die Unterstützung westlicher Experten zu diesem Zeitpunkt nicht möglich gewesen: "Had it not been for the special role of Martin and the Tongwenguan, Chinese access to international law would most probably have been postponed to a later phase of the modernisation of China."

Es ist diese Rekonstruktion der Übersetzung völkerrechtswissenschaftlicher Texte, die hier vor allem Anderen überzeugt. Entwickelt aus einer früheren Arbeit des Autors zur Übersetzung von Vattel (Svarverud 2000), beschreibt und kontextualisiert Svarveruds Buch die Übersetzungsprozesse klassischer Völkerrechtsautoren, wie etwa Wheaton, Charles de Martens, Bluntschli, Woolsey, Moynier, Hall und Phillimore. Besonders wertvoll, aus rechtshistorischer Perspektive, sind hier die Hinweise auf Übersetzungsschwierigkeiten einschlägiger juristischer Termini, für die es im klassischen Chinesisch keine Entsprechungen gab (»International Law «, »Law of Nature «, »right «, » sovereignty «, "neutrality «, » justice « etc.). Svarverud beschreibt anschaulich, wie und mit welchen Folgen diese Schwierigkeiten gelöst wurden, welche Neologismen sich bildeten, ob sie sich verstetigten oder wieder verschwanden. Abgerundet wird dieses Kapitel durch eine chronologische Bibliographie chinesischer Übersetzungen völkerrechtlicher Literatur zwischen I847 und I9II, die im Anhang des Bandes zu finden ist.

Die Übersetzung völkerrechtlicher Literatur aus westlichen Sprachen endete mit dem Krieg gegen Japan (I 894-95), in dem China auf dramatische Weise unterlegen war. Nach der Niederlage suchte die jüngere Generation chinesischer Wissenschaftler in großer Zahl den Weg 
nach Japan, um an den Universitäten des weiter entwickelten Nachbarn zu studieren. Dies blieb nicht ohne Auswirkungen auf den chinesischen Völkerrechtsdiskurs. Zwischen I 902 und I9I I basierten, wie Svarverud feststellt, beinahe alle völkerrechtlichen Publikationen in China auf japanischen Texten. Allerdings sollte dies nicht als ein Ende des Kulturtransfers aus dem Westen missverstanden werden. Japan selbst hatte sich seit der Meiji-Restauration (I 868) mit großem Erfolg dem westlichen Vorbild angenähert. Auch wenn Svarverud in Folge seiner theoretischen Annahmen nicht darauf hinweist, so offenbart die Orientierung an Japan letztlich wiederum die Bedeutung des westlichen Einflusses auf die Entwicklung des Völkerrechts in China.

An diesem Gesamteindruck ändert auch das abschließende Kapitel, welches der Autor selbst als den Kern seiner Arbeit begreift, nichts (I 87267). Dieses Kapitel enthält die Beschreibung des chinesischen Völkerrechtsdiskurses, wie er sich ab I 902 zunehmend intensivierte und schließlich über das Anfertigen von Übersetzungen und deren direkter Rezeption hinausging. Svarverud gibt hier einen interessanten und tiefen Einblick in chinesische Quellen, die eine vielschichtige Diskussion zeitgenössischer intellektueller Eliten über die Rolle Chinas in den internationalen Beziehungen widerspiegeln. Letztlich zeigen sich aber auch hier zunächst die westlichen Bezüge, aus denen heraus sich der lokale Diskurs entwickelte.

Wenn sich auch theoretische Rahmung und empirische Ergebnisse bei Svarverud in entscheidenden Punkten nicht entsprechen, so ist dieses Buch insgesamt dennoch ein wichtiger und erhellender Beitrag in der Analyse des interkulturellen Transfers von Recht. Gerade Svarveruds sprachwissenschaftliche Hinweise sind ein wesentlicher Beitrag zum Verständnis der Aneignung des Völkerrechts in China. »International Law as World Order in Late Imperial China « bietet für die Völkerrechtsgeschichte insofern inhaltlich wie methodisch interessantes Anschauungsmaterial.

\section{Mit dem Teufel aus einer Schüssel essen*}

Friedrich Gaus (I88I-I955) war Leiter der Rechtsabteilung des deutschen Auswärtigen Amtes von 1922 bis I943, ein juristischer Fachmann des Völkerrechts par excellence. Als promovierter Assessor war er 1907 in das Amt eingetreten, wo er vom Legationsrat zum Ministerialdirektor aufstieg. Bei allen wichtigen Verträgen Deutschlands war er dabei, von Brest-Litowsk bis Versailles, vom Schieds- und Vergleichsvertrag mit der Schweiz zum Vertrag von Rapallo, vor allem aber bei dem Vertrag von Locarno und bei Deutschlands Eintritt in den Völkerbund. Gaus galt als das juristische Gehirn der auswärtigen Politik. Er begleitete Stresemann bis zu dessen Tod 1929 und war bei seiner Diplomatie stets »der im Mittelpunkt stehende juristische Experte «. In dieser Funktion hatte er alle Minister und Staatssekretäre überlebt. Auch Stresemanns Nachfolger verzichteten nicht auf ihn und so kam er unter Minister Neurath ins Dritte Reich

\footnotetext{
Gerhard Stuby, Vom »Kron-

juristen « zum »Kronzeugen «.

Friedrich Wilhelm Gaus: ein

Leben im Auswärtigen Amt der

Wilhelmstraße, Hamburg: Verlag

für das Studium der Arbeiter-

bewegung (VSA) 2008, 5II S.,

ISBN 978-3-89965-284-0
}

Stefan Kroll 